\title{
Un atelier implicatif : « vivant » ou « non vivant »?
}

\section{Maryline Cantor}

\section{(2) OpenEdition}

1 Journals

Édition électronique

URL : http://journals.openedition.org/trema/2085

DOI : 10.4000/trema.2085

ISSN : 2107-0997

Éditeur

Faculté d'Éducation de l'université de Montpellier

\section{Édition imprimée}

Date de publication : 1 septembre 1996

Pagination : 169-176

ISSN : 1167-315X

\section{Référence électronique}

Maryline Cantor, « Un atelier implicatif : « vivant » ou « non vivant » ? », Tréma [En ligne], 9-10 | 1996,

mis en ligne le 18 septembre 2013, consulté le 01 mai 2019. URL : http://journals.openedition.org/ trema/2085; DOl : 10.4000/trema.2085

Ce document a été généré automatiquement le 1 mai 2019.

Trema 


\title{
Un atelier implicatif : « vivant » ou «non vivant »?
}

\author{
Maryline Cantor
}

\section{Introduction}

1 Les recherches sur les conceptions du vivant ont porté essentiellement sur celles présentes chez les enfants (Astolfi et al., 1972 ; Host et al., 1976 ; Lalanne, 1985). Diverses «recettes» sont possibles pour faire émerger ces conceptions initiales (De Vecchi, Giordan, 1989). Mettre les étudiants en activité présente l'avantage de pouvoir observer à la fois les compétences dans une démarche d'investigation et les conceptions «à l'œuvre ». L'atelier vécu à Montpellier est la réplique d'une situation de formation pour des enseignants de l'école élémentaire. Adapté d'un stage de professeurs en École Normale (coordonné par Jeannine Deunff), il est proposé aux étudiants de l'IUFM de Haute-Normandie par l'équipe de formateurs en Biologie (P. Bourgeois, M. Cantor, N. Drigout, J. Fihey, J.-M. Lange et I. Martinet). L'atelier propose tout d'abord une mise en activité puis un temps de « recul » et d'analyse de son déroulement. Il associe contenus scientifiques sur le concept de vivant et formation didactique sur les conceptions à l'œuvre et sur les démarches d'investigation. Il joue ainsi pleinement son rôle de formation pour les enseignants de l'école élémentaire (Antheaume, 1994 ; Cantor, 1994 ; Develay, 1994 ; Martinand, 1994). 
Anémia Salina. Cinq millimètres de long, foisonne dans les milieux aquatique salés, voire saumâtres.

À gauche : un spécimen juvénile, vue de côté. À droite : la poche d'œufs, à la queue. Image agrandie 25 fois.
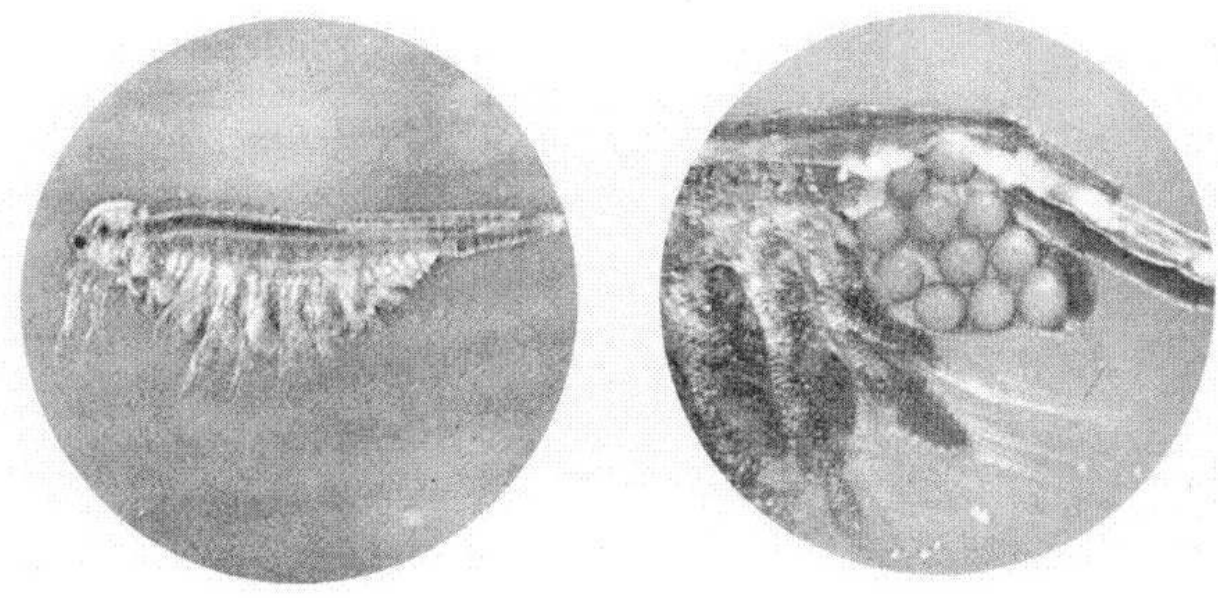

Ph. de : ZAHL P.A., "Great Salt Lake”, in Nat'l Geog. Mag., août, Vol. 132, №2, 1967.

\section{Présentation de l'atelier}

L'atelier se déroule sur une durée approximative de trois heures. La première moitié de la séance est consacrée à l'activité, ce qui nécessite de pouvoir facilement accéder à du matériel :

- des récipients (verres de montre, cuvettes, tubes à essai),

- du matériel d'observation simple (loupes à main),

- du matériel de chauffage simple et des pinces en bois,

- quelques outils de dissection (épingles, pinces fines),

- quelques colorants (eau iodée, bleu de méthylène, rouge neutre) et pipettes.

La deuxième moitié est réservée aux échanges entre étudiants et formateur et à l'analyse de la situation proposée.

\subsection{La mise en activité}

Des verres de montre numérotés contiennent des « objets » inconnus. La consigne est la suivante :

1. Dénombrez les « objets » différents.

2. Tentez d'identifier le contenu des verres de montre. Pour les « objets " que vous pensez avoir identifiés, cherchez un ou des moyens de prouver cette identité. Pour les autres, envisagez le ou les moyens de progresser dans leur approche.

3. Classez ces différents "objets" en vivant/non vivant (l'identité des «objets » est alors connue).

5 Aucune consigne précise concernant la démarche d'investigation n'est donnée. Du matériel, des instruments et des produits divers (voir ci-dessus) sont laissés en libre accès dans la salle. Le formateur précise que ce matériel peut être complété selon la demande. 
Les étudiants se répartissent en groupe de trois : deux sont des "investigateurs ", le troisième est un observateur » (rôle plus passif et plus difficile). L'observateur doit noter :

- les moyens d'investigation utilisés ;

- les démarches mises en œuvre ;

- les interactivités sociales ;

- les réactions affectives.

\subsection{Le temps du recul et de l'analyse}

Qu'y avait-il donc dans les verres de montre ? Chaque numéro correspond à un même contenu. Par exemple, à Montpellier, chaque équipe disposait de huit verres de montre.

D'autres contenus pourraient être proposés. Les critères de choix pour le formateur sont :

- la non toxicité ;

- une certaine analogie morphologique ;

- pour les vivants, l'exclusion de la mobilité qui représente une propriété trop visible (vie ralentie, œufs, graines,...).

\begin{tabular}{|c|l|}
\hline $\begin{array}{c}\text { No verre } \\
\text { de montre }\end{array}$ & \multicolumn{1}{c|}{ "objet » } \\
\hline No 1 & levure chimique (bicarbonate de soude) \\
No 2 & graines de pensée \\
\hline No 3 & sucre (saccharose) \\
No 4 & levure biologique \\
\hline No 5 & œufs d'Artémia salina \\
\hline No. 6 & œufs de Phasme (Carausius morosius) \\
No 7 & sel (chlorure de sodium) \\
\hline No 8 & talc non parfumé \\
\hline
\end{tabular}

Proposer des éléments provenant de vivants (petites coquilles, plumes ou poils, tests de radiolaires, ...) ou des restes fossilisés (fossiles de la craie : Globigèrines, Milioles ; sable fossilifère) se révèle également intéressant (questions et conceptions autour de "l'être vivant et le vivant ", de "vivant ou a été vivant", de "vivant ou a été fabriqué par un être vivant », de «traces, empreintes de vivant », ...). 


\section{Analyse du déroulement de l'atelier}

\subsection{Les consignes de l'activité}

Les consignes ne sont pas toujours respectées et le dénombrement d'« objets » n'est pas toujours fait. Des étudiants, séduits par le contenu des coupelles, se précipitent pour essayer de « dire ce qu'il y a dans le verre de montre ». Lors de la deuxième partie de la séance, l'analyse de l'intérêt de cette consigne montre la richesse des réflexions induites :

- contenu homogène ou mélange?

- nombre d'« objets » (nombre de grains, nombre d'œufs, de cristaux, ...) ou nombre de « catégories » (des graines, du sel, de la levure...).

\subsection{Les investigations}

Les « expériences » faites par les étudiants peuvent être comparées à celles effectuées par les enfants (Cauzinille-Marmeche et al., 1983 ; Astolfi et al., 1984).

11 Dans les moyens d'investigation, $90 \%$ des étudiants utilisent en premier une approche sensori-motrice. Beaucoup utilisent le goût et l'odorat, ce qui permet au formateur de faire une petite halte d'éducation à la sécurité » pour rappeler les précautions essentielles de préservation sensorielle face à des produits inconnus. Ce dernier précise alors qu'aucun des « objets » n'est toxique.

L'approche instrumentalisée, d'abord utilisée par quelques étudiants, diffuse ensuite très vite dans l'ensemble du groupe (loupe à mains, essais de dissection, ...).

13 Pour $90 \%$ des étudiants, la démarche reste tâtonnante, avec interrogation sur le contenu des différents verres de montre séparément. Certains étudiants (et plutôt ceux qui ont eu une formation initiale en sciences expérimentales) mettent en place une investigation avec une observation comparative des différents « objets »ou des éléments de protocole de tests systématiques (action de la chaleur par exemple).

Pour les contenus que les étudiants pensent avoir identifié, il y a souvent recherche d'une seule « preuve » : "C'est du sucre parce que c'est sucré quand on le goûte ! »

Des tests de «mise en réfutation » sont parfois recherchés spontanément : "Ça doit être de la farine,... Ah non, ça ne brûle pas ! », « Ce n'est pas de la poudre de craie car il n'y a pas effervescence à l'acide. »

16 Pour les "objets non identifiés ", peu d'étudiants mettent en place un questionnement systématique pour effectuer les tests. La majorité font une approche tâtonnante, avec des a priori successifs sur la nature du contenu de la coupelle. Il y a comparaison avec des objets connus, avec des pré-acquis mais ces comparaisons restent non organisées et simplement en relation avec des connaissances factuelles du quotidien : "C'est peut-être du talc,... Au toucher, ça ressemble, mais comment être sûr? ». Dans cet exemple, le talc, les œufs d'artémie et les œufs de phasme ont suscité le questionnement le plus important. 


\subsection{Le moment de « recul » et d'analyse}

17 Ce moment, très important, permet tout d'abord la confrontation des investigations et la réflexion autour de la notion de «preuve ».

- Des œufs de phasmes peuvent avoir été mis à germer car ils ressemblent à des "graines ». "Mais quand on ouvre on voit que c'est assez liquide, ce n'est pas sec comme des graines », rétorquent souvent des étudiants.

- Les œufs d'artémie demeurent un mystère (sauf pour les étudiants amateurs d'aquariophilie ou pour les anciens lecteurs de Pif Gadget !). « Ce n'est pas du poivre, j'ai goûté ! ». « Ça carbonise quand on le brûle, ça a une origine organique! ». Sans information ou documentation supplémentaire, il est difficile de trouver « spontanément » les conditions naturelles de développement de ces œufs. Si des œufs ont été mis en éclosion dans un peu d'eau salée quelques jours auparavant, les étudiants peuvent observer les petits crustacés à la loupe binoculaire.

Extrait de manuel d'élève, Science et Technologie. p. 68 : partie encyclopédique, unité du vivant. CANTOR \& SCHNEEBERGER, Manuel d'élève : Sciences et Technologie, Cycle 3, niveau 1, coll. Gulliver, Paris, Nathan, 1995.

\section{Les êtres vivants?}

Deux biologistes écrivent. A la sortie du Moyen-Âge, au siècle des ingénieurs, certains se représentaient les animaux comme des machines, des mécaniques, des automates. Selon André Langaney, un automate et un être vivant ont des relations différentes avec leur environnement. Par exemple, Robox n'est pas capable d'inventer un nouveau comportement.

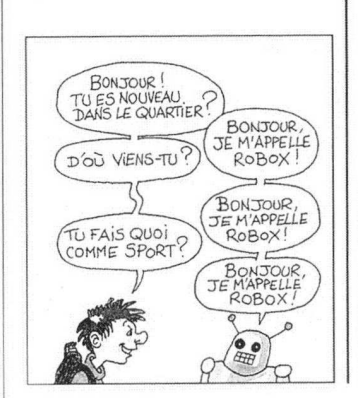

La diversité du monde vivant est extraor-

dinaire : le chêne et le roseau, le rat, la

belette et le petit lapin, le cèpe et les

moisissures, les algues... Qui ne les a pas

vus?

On dénombre actuellement plus de deux millions d'espèces différentes auxquelles s'ajoutent environ deux milliards

d'espèces qui ont existé et qui n'existent plus.

Qu'y a-t-il de commun entre tous ces êtres vivants? Tous les êtres vivants se reconstruisent eux-mêmes, à tout moment, à partir d'éléments qui leur sont étrangers. C'est ainsi qu'une carotte fait une carotte avec de l'eau, de l'air, les sels du terrain où elle a été plantée et du soleil

C'est ainsi qu'un lapin fait du lapin avec des carottes (d'après Jean Tavlitzki)

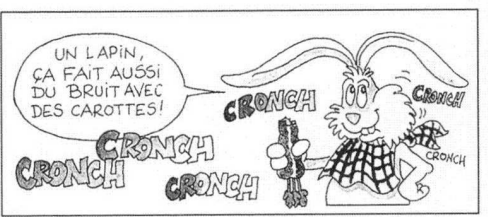

La mise en commun des observations de la situation et des réactions qu'elle a provoquées facilite ensuite la réflexion sur les conceptions du vivant. Elle sollicite un questionnement supplémentaire et des apports d'informations scientifiques 
sur le concept de vivant (Jacob, 1970 ; Deunff, 1989 ; Turquier, 1989 ; Rumelhard, 1995) et didactiques sur les conceptions du vivant des enfants (Host et al., 1976).

La discussion permet, en outre, des constats sur les activités et les pratiques expérimentales :

- les démarches d'investigation utilisées spontanément, le tâtonnement expérimental, les limites des sens (les édulcorants), l'observation (on ne voit bien que si on sait et si on reconnaît, le rôle de l'imagination, ...),

- l'importance des interactions sociales lors de l'activité (le travail dans l'équipe, les échanges entre équipes, le rôle de la contradiction, les effets d'imitation, ...),

- les attitudes développées (la curiosité, le bon sens, le sens de l'organisation, ...),

- les réactions affectives à surmonter (la peur, le dégoût postérieur quand on sait ce que l'on a goûté, ...).

Extrait de manuel d'élève, Science et Technologie, p. 9 : partie documents, unité du vivant. CANTOR \& SCHNEEBERGER, Manuel d'élève : Sciences et Technologie, Cycle 3, niveau 1, coll. Gulliver, Paris, Nathan, 1995.
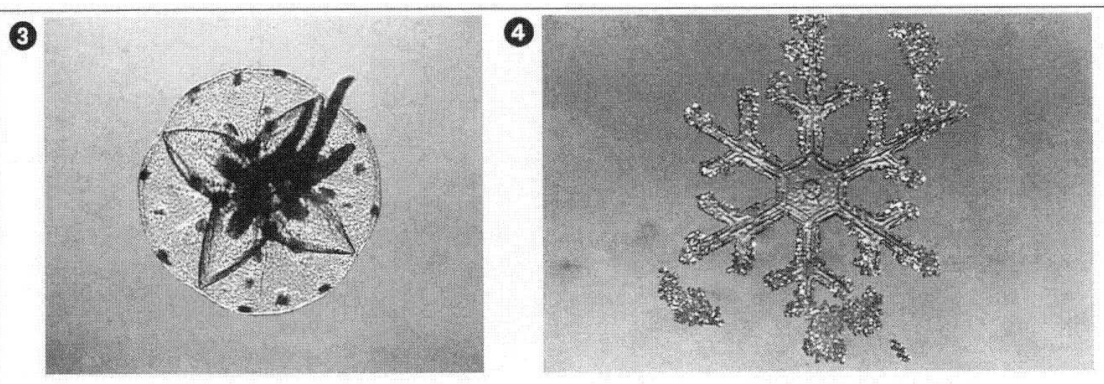

Deux photos : sur l'une, un cristal de neige, sur l'autre une petite méduse. Il semble y avoir des ressemblances...

D'après toi, quel est le cristal de neige ?

L'un est vivant, l'autre non.

Quelles sont les différences? Comment savoir si ce que tu rencontreras au cours de tes découvertes est vivant ou non? Quelles questions te poseras-tu ?

\section{Discussion}

La discussion autour de cet atelier (rapporteur Guy Rumelhard) s'est engagée sur le sens du travail et sur les diverses consignes possibles à partir de la même situation :

- «Distinguer ce qui est vivant et non vivant».

21 Mais ici, il se pose plusieurs problèmes. Suffit-il de retenir un critère (une propriété) positif (croissance, mobilité...) ou négatif (cristallisation)? Ou bien faut-il plusieurs critères présents simultanément? Ici, de toute évidence on masque l'une des propriétés du vivant (" qui bouge par soi-même ») et qui est bien souvent suffisante, même si elle ne s'applique pas de manière systématique. On est obligé de faire intervenir plusieurs critères simultanément (morphologie, composition chimique,...) et surtout, 1 faut faire intervenir le facteur temps (il faut mettre en développement les œufs et les graines pour confirmer qu'ils sont encore vivants).

- «Proposer un minimum de critères pour distinguer les différents « objets » (on s'arrête quand tout est distingué). « En trouver un seul pour les séparer tous ». 
- «Trouver tous les critères possibles ». « Donner une définition (une caractérisation) la plus complète possible compte tenu des éléments d'investigation disponibles».

- Laisser faire de manière empirique, sans consigne, et observer ce que produisent « spontanément » les étudiants.

- Donner a priori une définition complète du vivant/non vivant et l'appliquer à la situation (reste à savoir quelle(s) définition(s)!).

\section{Conclusion}

L'atelier se déroule en début d'année universitaire. Il a un impact positif, aussi bien sur les objectifs de formation que pour la mise en confiance et en cohésion du groupe d'étudiants. Il s'agit d'une première approche des différentes questions et d'une "déstabilisation" concernant leurs conceptions sur le vivant et sur la démarche scientifique. D'autres mises en situations, avec alternance d'activités (en particulier recueil de conceptions d'enfants sur le vivant lors du stage en classe de maternelle), analyse de documents (scientifiques ou didactiques) et apport d'informations (Cantor, Schneeberger, 1995 ; Cantor, Giordan, 1996) fournissent un environnement didactique (De Vecchi, Giordan, 1989) pour aider les étudiants à évoluer dans leur formation.

BURGESS J., MARTEN M. \& TAYLOR R., Microscopie - Explorer l'Invisible, Paris, Larousse, 1987, Réédition de 1991.

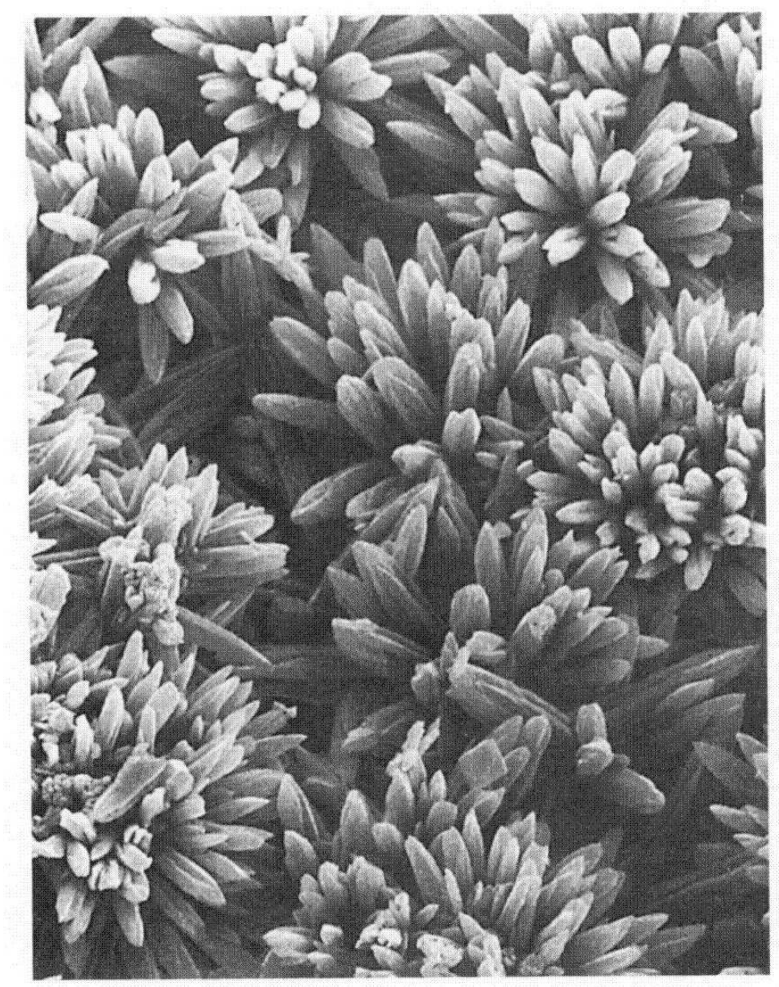




\section{BIBLIOGRAPHIE}

ANTHEAUME P., «Situations didactiques ponctuelles et formation professionnelle », in Sciences et techniques, quelles activités? Quels acteurs ?, Créteil, IUFM, 1994, p. 10-27.

ASTOLFI J.-P., COULIBALY A. \& HOST V., Biologie (initiation expérimentale) en $6^{e}$ et $5^{e}$, Coll.

Recherches pédagogiques, Paris, INRP, 1972, p. 55.

ASTOLFI J.-P., CAUZINILLE E., GIORDAN A., HENRIQUES A., MATHIEU J. \& WEIL-BARAIS A., Expérimenter : sur les chemins de l'explication scientifique, Toulouse, Privat, 1984.

CANTOR M., « Conceptions des apprenants et formation des élèves-instituteurs ", 1994, in :

Cantor M., Schneeberger P. et al, (coord. : Astolfi J.-P.), Sciences et Technologie, manuel élève, cycle 3. niveau 1, coll. Gulliver, Paris, Nathan, 1995.

CANTOR M., SCHNEEBERGER P. et al., in Sciences et Technologie, guide pédagogique, cycle 3, niveau 1, coll. Gulliver, (coord. : Astolfi, J.-P.), Paris, Nathan, 1995.

CANTOR M., GIORDAN A. et al., Les sciences et la technologie à l'école maternelle : comment faire ?, Nice, Z'éditions, 1996.

CAUZINILLE-MARMECHE E., MATHIEU J., WEIL-BARAIS A., Les savants en herbe, Berne, Peter Lang, 1983.

CLÉMENT P., « De la régulation à l'auto-organisation », in La régulation en biologie. Approche didactique : représentation, conceptualisation, modélisation, (coord. : Rumelhard G.), Paris, INRP, coll. Didactiques des Disciplines, 1995, p. 7-24.

COLLECTIF, Éveil scientifique et modes de communication, Paris, INRP, Coll. Recherches pédagogiques, 117 p., 1983.

DE BUEGER-VANDER BORGHT C. (s.p.), «Observer scientifiquement », septièmes rencontres européennes de didactique de la Biologie, Montpellier, 1995.

DEUNFF J., « Réseau des concepts essentiels "La trame conceptuelle" », in Échanges (revuede l'association nationale des conseillers pédagogiques), $\mathrm{N}^{\circ} 20$, Chatellerault, France, 1989, p. 9-18.

DE VECCHI G. \&. GIORDAN A., L'enseignement scientifique : comment faire pour que « ça marche »?, Nice, Z'éditions, 1989.

DEVELAY M., Peut-on former les enseignants ?, Paris, ESF éditeur, Coll. Pédagogies, 1994.

GIORDAN A., GIRAULT Y. \& CLEMENT P. (coord.), Conceptions et Connaissances, Berne, Suisse, Peter Lang, p. 147-156.

GOHAU G., « Esprit déductif versus esprit inductif », in Aster, N 14, p. 9-20, Paris, INRP, 1992.

HOST V. et al., Activités d'éveil scientifiques, 4 : Initiation biologique, Paris, INRP, Coll. Recherches pédagogiques, 1976, p. 86.

JACOB F., La logique du vivant, Paris, Gallimard, 1970, (réed., TEL Gallimard, 1983).

KERLAN A., ROSSI C., COTTET-EMARD G. \& VERIN A., Évaluer pour former, Paris, INRP, Coll. rencontres pédagogiques, $\mathrm{N}^{\circ} 22,1988$. 
LALANNE J., « Le développement de la pensée scientifique (orientation biologique) chez les enfants de 6 à 14 ans ", in Aster, N 1, Paris, INRP, 1985.

MARTINAND J.-L., « Observer-Agir-Critiquer- L'enseignement des sciences expérimentales à l'école élémentaire ", in Actes des Journées Langevin, Brest, 7 p., 1994.

RUMELHARD G., « De la biologie contemporaine à son enseignement », in Develay M. (dir.), Savoirs scolaires et didactiques des disciplines : une encyclopédie pour aujourd'hui, Paris, ESF, 1995, p. 317-337.

TURQUIER $\mathrm{M}^{\mathrm{me}}$, « La notion d'être vivant (nouvelles approches), » in Échanges (revue de l'association nationale des conseillers pédagogiques), France, Chatellerault, $N^{\circ} 20,1989$, p. 19-28.

\section{RÉSUMÉS}

Cet atelier est proposé à des élèves - professeurs des écoles étudiant en IUFM. Pour identifier des objets inconnus et les classer en vivant " ou "non - vivant ", les étudiants mettent en œuvre diverses démarches. Cette activité fonctionnelle facilite l'émergence de leurs conceptions sur le vivant. Elle permet ensuite de comparer les démarches d'investigation spontanément utilisées. La mise en commun des observations et l'analyse de ce que les étudiants ont vécu lors de l'atelier représentent une situation de formation professionnelle de grand intérêt.

This workshop is proposed to "IUFM" student teachers aspiring to teach grade school. In order to identify unknown objects and to classify them as either "living" or "non-living" the student teachers put into practice various methods. This functional activity facilitates the emergence of their conceptions about living things. It afterwards allows them to compare the investigative steps they spontaneously took. Bringing together each and every student's observations as well as the analysis of what they experienced during the workshop both represent a highly profitable situation from a professional training standpoint.

\section{INDEX}

Mots-clés : conception sur le vivant, démarche d'investigation, situation de formation Keywords : investigative approach, professional training learning-situation, theoretical viewpoint on living thing

\section{AUTEUR}

\section{MARYLINE CANTOR}

IUFM de Haute-Normandie et université de Rouen, laboratoire LIREST/GDSTC, ENS Cachan 